\title{
PSICOLOGÍA POSITIVA, EDUCACIÓN EMOCIONAL Y EL PROGRAMA AULAS FELICES
}

\section{POSITIVE PSYCHOLOGY, EMOTIONAL EDUCATION AND THE HAPPY CLASSROOMS PROGRAM}

\author{
Rafael Bisquerra Alzina' y Silvia Hernández Paniello \\ 'Universidad de Barcelona. ${ }^{2}$ CIFE (Centro de Innovación y Formación Educativa) Juan de Lanuza de Zaragoza
}

La psicología positiva tiene múltiples aplicaciones. En este artículo nos centramos en la educación formal, de los 3 a los 18 años. Se expone como el desarrollo del bienestar debería ser una de las finalidades de la educación, lo cual repercutiría en el profesorado, alumnado, familias y por extensión a la sociedad en general. La educación emocional (Bisquerra, 2000, 2009), desde sus orígenes, ha tenido esto claro. Con el surgimiento de la psicología positiva se produjo un renovado esfuerzo en este sentido, como una mejor fundamentación. El GROP (Grup de Recerca en Orientación Psicopedagógica) de la Universidad de Barcelona está realizando investigaciones en esta línea. Aulas Felices es el primer programa en lengua castellana de psicología positiva aplicada a la educación; dirigido a alumnado de educación infantil, primaria y secundaria. El programa centra sus aplicaciones en las fortalezas personales y la atención plena; es gratuito y de libre distribución. En el artículo se argumenta sobre la importancia de potenciar el bienestar en la educación, se presentan actividades prácticas y estrategias de intervención, con especial referencia a la formación del profesorado.

Palabras clave: Psicología positiva, Bienestar, Educación emocional, Fortalezas personales, Atención plena.

Positive psychology has multiple applications. This article is focused on formal education, from the ages of 3 to 18 years. The development of well-being should be one of the aims of education, which would affect teachers, students, families and by extension society at large. This has been a clear aim for emotional education (Bisquerra, 2000, 2009), from the outset. With the emergence of positive psychology, there was a renewed effort in this direction, as a means of providing a better foundation. The GROP (Grup de Recerca en Orientación Psicopedagógica [Research in Psychopedagogical Education Group]) at the University of Barcelona is conducting research on this subject. The Happy Classrooms ("Aulas felices") program developed by the SATI Team is the first program in Spanish aimed at working on positive education. It is designed for children and youths in pre-school, primary and secondary education. The program focuses its applications on character strengths and mindfulness. It is freely available for access and distribution. This article argues for the importance of enhancing well-being in education. Practical activities and intervention strategies are presented, with special reference to the importance of teacher training.

Key words: Positive psychology, Well-being, Emotional education, Character strengths, Mindfulness.

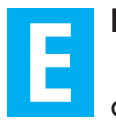

\section{BIENESTAR COMO META DE LA EDUCACIÓN}

El bienestar debería ser una de las metas importantes de la educación y de la políitica. Pero ¿̇de qué bienestar estamos hablando? En los medios de comunicación, cuando se habla de bienestar se sobrentiende el bienestar material, entendido como el desarrollo económico y tecnológico. Aquí nos referimos a otro tipo de bienestar. La diversidad de enfoques sobre el bienestar justifica la conveniencia de "poner un apellido" para concretar de qué bienestar hablamos: bienestar material, bienestar social, bienestar físico, bienestar profesional (engagement), bienestar emocional, bienestar subjetivo, bienestar psicológico, bienestar hedónico, bienestar eudemónico (Bisquerra, 2013). Todos estos tipos de bienestar deberían formar parte de la educación, ya que nos orientan en el camino a seguir.

Conviene dejar claro que el bienestar tiene una dimensión personal y otra social. Hay que trascender esta visión miope del bienestar personal individual para orientarnos hacia el de-

Recibido: 15 Febrero 2016 - Aceptado: 6 Mayo 2016

Correspondencia: Rafael Bisquerra. Universitat de Barcelona (UB). Campus Mundet, Edifici Llevant. Passeo Vall d'Hebron, 171.08035 Barcelona.España.E-mail: rbisquerra@ub.es sarrollo integral de las personas dentro de sus respectivas organizaciones. El objetivo es el bienestar social en interacción con el bienestar personal.

No hay que confundir educación con escolarización. Escolarización equivale a educación formal y se circunscribe a los centros de educación infantil, primaria y secundaria. Pero la educación es un concepto mucho más amplio que se refiere a cualquier proceso de aprendizaje en cualquier contexto, y a lo largo de la vida. Si bien, en este artículo nos vamos a ceñir a la educación formal.

Conviene subrayar que los centros educativos son el medio idóneo para la difusión de la cultura del bienestar. Entre otras razones porque de esta forma se empieza desde las primeras edades y también porque es el modo más efectivo de llegar a la totalidad de la población. En efecto, en los países desarrollados casi el cien por cien de los niños pasa por los centros educativos y a través de estos centros se tiene acceso a las familias. Por tanto, la intervención a través de la educación formal asegura el acceso a gran parte de la población. No hay otra intervención que pueda asegurar mayor alcance. 


\section{EDUCACIÓN EMOCIONAL PARA EL BIENESTAR}

La educación emocional tiene como objetivo el desarrollo de las competencias emocionales y el bienestar (Bisquerra, 2000, 2009). Se basa en el principio de que el bienestar es uno de los objetivos básicos de la vida personal y social. La felicidad muchas veces se busca por caminos equivocados, que pueden conducir a comportamientos de riesgo, como consumo de drogas. La educación para el bienestar basado en la ciencia debe contemplarse como esencial para el desarrollo integral del alumnado.

La psicología positiva, la inteligencia emocional y la educación emocional, aportan evidencias de lo que funciona y lo que no. La inteligencia emocional es la capacidad (hability) para percibir y expresar las emociones, utilizar las emociones para facilitar el pensamiento, comprender y razonar a través de las emociones y regular las emociones en uno mismo y en los demás (Mayer, Salovey, Caruso y Cherkasskiy, 2015). Los conocimientos de la psicología positiva y la inteligencia emocional deben ser difundidos a través de la educación emocional, con el objetivo de desarrollar competencias clave para la vida que permitan alcanzar un mayor bienestar.

La educación emocional debe iniciarse en los primeros momentos de la vida y debe estar presente a lo largo de todo el ciclo vital.

La educación emocional sigue una metodología eminentemente práctica (dinámica de grupos, autorreflexión, razón dialógica, juegos, relajación, respiración, etc.) con objeto de favorecer el desarrollo de las competencias emocionales.

En general, el profesorado no ha recibido una formación inicial o continua en educación emocional y son los primeros que la necesitan para poder contribuir al desarrollo de competencias emocionales en el alumnado. En este sentido se puede afirmar que el profesorado y las familias son los primeros destinatarios de la educación emocional.

Con motivo de cumplirse los 25 años de la publicación del primer artículo sobre inteligencia emocional (Mayer y Salovey, 1990), se ha elaborado una revisión de las investigaciones con aplicaciones a la educación (Bisquerra, García Navarro y PérezGonzález 2014) de donde se derivan principios para la implantación de programas.

\section{APLICACIONES DE LA PSICOLOGÍA POSITIVA A LA EDUCACIÓN}

La psicología positiva es una corriente que surge en el cambio de siglo y que experimenta una considerable difusión desde los primeros momentos, como si se tratase de un cambio de paradigma que abre nuevas esperanzas hacia un futuro mejor, centrado en el bienestar de las personas y de la sociedad en general. Esto tiene unas aplicaciones muy importantes en la educación.

La psicología positiva se propone como pilares básicos de estudio e investigación: las emociones positivas, los rasgos in- dividuales positivos (virtudes y fortalezas), las instituciones positivas que facilitan su desarrollo y los programas que ayudan a mejorar la calidad de vida de las personas y previenen la incidencia de psicopatologías.

Para Linley, Harrington, Stephen, y Wood (2006) la psicología positiva es el estudio científico del funcionamiento humano óptimo. En un nivel metapsicológico, pretende compensar el desequilibrio en la investigación y la práctica psicológica llamando la atención acerca de los aspectos positivos del funcionamiento y la experiencia humanos, e integrándolos dentro de nuestra comprensión de los aspectos negativos del funcionamiento y la experiencia humanos. En un nivel pragmático, trata acerca de la comprensión de las fuentes, los procesos y los mecanismos que conducen a éxitos deseables.

Como consecuencia de las investigaciones en psicología positiva, los profesionales están en mejores situaciones para ayudar a las personas a construir su bienestar y florecer (flourish) más que simplemente existir. Esto significa mejorar la calidad de vida y el bienestar subjetivo; prevenir la aparición de trastornos mentales y psicopatologías; desarrollar competencias emocionales, etc. Todo ello sin apartarse de la más rigurosa metodología científica.

Los temas habituales de la psicología positiva son el bienestar, las fortalezas humanas, el fluir, el optimismo, el humor, la creatividad, la resiliencia, la inteligencia emocional, la atención plena o mindfulness, etc. Todos estos contenidos deberían formar parte del currículum académico obligatorio de los estudiantes de primaria y secundaria, así como de la Universidad.

\section{LA EDUCACIÓN EMOCIONAL PARA EL BIENESTAR EN EL GROP}

El GROP (Grup de Recerca en Orientación Psicopedagógica) de la Universidad de Barcelona (UB) se fundó en 1997 con la intención de investigar sobre educación emocional. Se formuló un modelo de competencias emocionales, que está en revisión permanente, y que incluye cinco bloques: conciencia emocional, regulación emocional, autonomía emocional, competencias sociales, habilidades de vida para el bienestar (Bisquerra, 2009).

Cuando el GROP llevaba tres años trabajando, surgió la psicología positiva, que dio un gran impulso al quinto bloque antes señalado (habilidades de vida para el bienestar). En el 2002 se creó en la UB el Postgrado en Educación emocional y bienestar, que tiene entre sus fundamentos a la inteligencia emocional, la neurociencia y la psicología positiva, entre otros. De hecho, el desarrollo del bienestar consciente es uno de los objetivos esenciales de la labor del GROP.

Los estudiantes del postgrado realizan unas prácticas en centros educativos que en general consisten en el diseño, aplicación y evaluación de programas de educación emocional, en los cuales, lógicamente, el bienestar es un aspecto esencial. Está en proceso de realización una evaluación de los efectos de 
todo esto en la práctica educativa. De momento, por las evidencias de que se dispone, se puede afirmar que el profesorado implicado manifiesta una clara satisfacción por las mejoras en competencias emocionales, reducción de la conflictividad y violencia, mejora del clima de aula, e incluso mejora del rendimiento académico.

La educación emocional se puede aplicar en todas las materias académicas como si fuera un tema transversal. Si bien, la tutoría ofrece un espacio idóneo para las prácticas (Bisquerra, 2012). Ejemplos de actividades son señalar en paneles la emoción que experimentan con diferentes expresiones faciales, expresar o verbalizar las emociones positivas que "sienten", agradecer las cosas buenas que les ocurren a través de textos o de modo oral, dedicar los primeros o últimos cinco minutos del día a comentar aspectos positivos de la vida, realizar prácticas de relajación y meditación en algunos momentos de la jornada escolar.

Las actividades escolares pueden ser divertidas, porque la diversión no es lo contrario de lo serio, sino de lo aburrido. Todavía tenemos el reto de pasar de una escuela basada en "la letra con sangre entra" a una escuela donde el lema sea "enseñar deleitando". El valor del juego para el aprendizaje debería ser redescubierto para hacer las actividades académicas divertidas.

El sentido del humor es un paso más de lo anterior. El humor no tiene por qué disminuir la disciplina, sino que puede aumentar el respeto hacia el profesor. Hay que aceptar que el aprendizaje supone esfuerzo y sacrificio; pero esto no es incompatible con el humor y la diversión.

Crear un clima de seguridad donde el alumnado se sienta cómodo, tanto con los compañeros como con el profesorado. El miedo está en el otro extremo de la felicidad. Un clima de miedo originado por causas diversas: miedo a los exámenes, al profesor, a los castigos, miedo a los compañeros (bullying), etc. Conviene vigilar y tener sensibilidad para contrarrestar los miedos en un clima de seguridad.

Las actividades grupales propician el bienestar. Las investigaciones han puesto de manifiesto que uno de los factores principales de bienestar son las relaciones sociales. Por tanto, realizar dinámicas de grupo en clase puede favorecer el aprendizaje y el bienestar. Algunas metodologías como el aprendizaje cooperativo también favorecen las relaciones sociales.

Con la intención de poner al alcance del profesorado interesado actividades, ejercicios y recursos, se han elaborado diversos materiales por parte de miembros del GROP. Algunos ejemplos son los siguientes. López $(2003,2011)$ ha elaborado materiales y recursos para la educación infantil; Renom (2003) para la educación primaria; Pascual y Cuadrado (2001) para la ESO; Güell y Muñoz (2003) para la secundaria postobligatoria; Agulló et al. (2010) han presentado recursos para la práctica de la educación emocional en general; Álvarez
(2001) ha desarrollado la metodología del diseño y evaluación de programas de educación emocional; Bisquerra (2011) presenta propuestas para educadores y familias; diversos miembros del GROP (2009) actividades para el desarrollo de la inteligencia emocional en los niños; Redorta et al. (2006) la relación entre emoción y conflicto; Bisquerra (2008) expone el enfoque de la educación emocional en la educación para la ciudadanía; Soldevila (2009) la educación emocional en las personas mayores; Bisquerra et al. (2014) presentan propuestas para la prevención del acoso escolar a través de la educación emocional; Filella (2014), la aplicación de la educación emocional a través de videojuegos; Pujol y Bisquerra (2012) elaboran un material didáctico para niños titulado El gran libro de las emociones; Bisquerra (2015) presenta un material didáctico denominado Universo de emociones, etc.

El diseño, aplicación y evaluación de programas de educación emocional es una de las líneas de trabajo del GROP, sobre la cual se han presentado más de diez tesis doctorales y multitud de artículos en revistas. Los resultados en general son altamente positivos. Para más detalles sobre las actividades, investigaciones y publicaciones del GROP, se puede visitar la web: http://www.ub.edu/grop/castellano/, donde se puede consultar directamente abundante bibliografía sobre el tema.

\section{LA PSICOLOGÍA POSITIVA EN EL PROGRAMA AULAS FELICES}

El programa "Aulas Felices" es elaborado por el Equipo SATI formado por Ricardo Arguís, Ana Bolsas, Silvia Hernández y Mar Salvador. La palabra sati procede de la antigua lengua pali, cuya traducción aproximada es atencion plena. El equipo está compuesto por cuatro docentes que trabajan en diferentes etapas educativas, con una ámplia y variada trayectoria laboral y constituído con el deseo de trabajar la psicología positiva en el ámbito educativo.

Tras conocer la psicología positiva, observar sus posibilidades de aplicación a los centros educativos y rastrear en el mercado editorial e internet las propuestas que existían sobre el tema, deciden crear un programa educativo que haga compatibles el aprendizaje de los contenidos escolares con el bienestar en las aulas. Sus objetivos principales son dos: incrementar la felicidad en alumnado, profesorado y familias y potenciar el desarrollo personal y social del alumnado.

Aulas Felices se publica en Internet en octubre de 2010. En noviembre de 2012 sale la segunda edición revisada y mejorada. Este programa toma como marco conceptual las aportaciones científicas de la psicología positiva. Ofrece unas 300 actividades, sugerencias metodológicas y propuestas generales de actuación coherentes con el programa.

Está dirigido a alumnado de 3 a 18 años. Las actividades para los cursos superiores también se pueden utilizar con personas adultas. Puede aplicarse en todas las áreas curriculares; también desde la acción tutorial y la educación en valores. El programa se sitúa en el marco de las competencias clave de 
los actuales sistemas educativos europeos; permite al profesorado trabajar las competencias más transversales: sentido de iniciativa y espíritu emprendedor, competencia social y cívica, competencia para aprender a aprender.

Se trata de un programa de libre distribución que puede descargarse gratuitamente en www.aulasfelices.org. También está disponible la versión en inglés. Su generosa difusión a través de la red y el trabajo concienzudo que hay tras el material hace que tenga el reconocimiento por parte del profesorado que lo conoce. Actualmente se aplica en unos 100 centros educativos. Es lectura recomendada en programas universitarios, masters y doctorado de diversas universidades españolas y extranjeras.

El Equipo SATI parte del modelo de bienestar descrito por Seligman (2002: 346-347; 2011 ) conocido como PERMA. La denominación es un acróstico de sus cinco componentes:

Positive emotions ("vida placentera")

Engagement ("vida comprometida").

Relationschips (relaciones sociales).

Meaning ("vida significativa").

Achievements ("logros").

Aulas Felices toma en consideración las investigaciones de la psicología positiva en cuanto aportan evidencias de que para ser feliz conviene desarrollar fortalezas personales y un estado de serenidad que ayude a alcanzar satisfacción en la vida. Se unen así, felicidad y perfeccionamiento humano. El bienestar no depende tanto de circunstancias externas sino de cómo las percibimos y afrontamos.

\section{Características básicas}

Las características básicas del programa son las siguientes:

$\checkmark$ Un modelo integrador que permite potenciar el desarrollo personal y social del alumnado.

- Unifica y estructura el trabajo que, en la actualidad, se está desarrollando en las escuelas en torno a la acción tutorial, la educación en valores y las competencias clave relacionadas con el desarrollo personal, social y el aprender a aprender.

$\checkmark$ Un conjunto de recursos amplio y flexible aplicable con alumnos de 3 a 18 años, con posibilidad de aplicar a todas las áreas y en todas las situaciones escolares sin tener la sensación de ser algo añadido.

$\checkmark$ Tiene dos ejes, que son dos conceptos de especial relevancia dentro de la psicología positiva: la atención plena y la educación de las 24 fortalezas personales (Peterson y Seligman, 2004). Todo esto con el referente final de hacer al alumnado más autónomo, más capaces de desenvolverse en el mundo que les rodea $y$, en definitiva, más felices" (Equipo SATI, 2012, p. 11).

En coherencia con las premisas de la psicología positiva y con el rigor con que se creó el programa, están en marcha varias investigaciones para obtener datos sobre la eficacia del programa, cuyos resultados estarán pronto disponibles.

\section{ATENCIÓN PLENA}

La atención plena o "mindfulness" es una actitud permanente de consciencia y calma que nos permite vivir íntegramente en el momento presente. Sus raíces se remontan a antiguas tradiciones orientales, siendo investigado y promovido en años recientes desde la psicología moderna. Es importante subrayar que la atención plena no se limita a técnicas de relajación y concentración. Va más allá, ya que integradas en la práctica regular, son el germen de una actitud vital, una filosofía de vida que permite vivir de modo más profundo cada momento, y con más disfrute.

En la actualidad, la práctica de la atención plena está siendo promovida por psicólogos, médicos y educadores en diversos países y en múltiples contextos profesionales, y sus beneficios están siendo respaldados por un creciente número de investigaciones (Brown y Ryan, 2003; Cebolla y Miró, 2008; Davidson y Begley; 2012; Kabat-Zinn, 1990, 2005; Lavilla, Molina y López, 2008; Lyubomirsky, 2008; Simón, 2007 y Vallejo, 2006 ;). De las cuales se desprende que "la práctica de la atención plena puede producir numerosos efectos sumamente beneficiosos, entre los que destacan:

$\checkmark$ Aumentar la concentración.

$\checkmark$ Reducir automatismos.

$\checkmark$ Lograr un mejor control de pensamientos, emociones y conductas.

$\checkmark$ Disfrutar más del momento presenten

$\checkmark$ Efectos físicos saludables: relajación, mejora de la respiración, regulación de la presión arterial, potenciación del sistema inmunitario...

- Cambios positivos a nivel neurobiológico" (Equipo SATI 2012, p. 37)

Los avances en psicología y el apoyo científico a la práctica de la atención plena hacen que su aplicación al contexto escolar se incremente y sea cada vez más reconocida. Arguís (2014) argumenta la importancia de la atención plena en el ámbito educativo. Según el autor, "el mindfulness ayuda a:

v Contrarrestar el estrés diario, para vivir las experiencias de un modo más atento y consciente.

$\checkmark$ Asimilar las informaciones que recibimos de forma más serena y relajada, para aprender mejor y evitar la "infoxicación".

$\checkmark$ Aportar elementos para el desarrollo personal y social y la promoción del bienestar en las escuelas, que complementen los aprendizajes académicos tradicionales" (p. 131).

Los docentes antes de realizar esta actividad en el aula, deben formarse y ejercitar la atención plena para trabajar de modo adecuado con el alumnado y aprovechar igualmente las ventajas de la práctica sistemática.

Para entrenar al alumnado en la atención plena, existen diversas técnicas como las siguientes. Una descripción más detallada puede encontrarse en el capítulo 7 del programa Aulas Felices. 
$\checkmark$ Meditación basada en la respiración, respiración caminando.

$\checkmark$ écnicas basadas en la relajación y meditación: body Scan, ejercicios derivados del yoga, tai chi, chi kung.

$\checkmark$ La atención plena en la vida cotidiana: saborear con calma un alimento; disfrutar de una imagen, una música; seleccionar tareas cotidianas y realizarlas con plena consciencia, etc.

Por ejemplo, la actividad "Mis manos (I): la caricia". Tiene el objetivo de favorecer la conciencia sensorial y la concentración. Se puede resumir así: Tras realizar unas respiraciones tranquilas, el alumnado observa sus manos; las junta por las palmas, intentando acercarlas al máximo presionándolas; se sueltan dejando únicamente las puntas de los dedos en contacto. Se repite varias veces. Tras separar las manos, se cierran y abren simultáneamente, notando la presión suave de las uñas en la piel al cerrar el puño y su estiramiento al abrirlas. Para terminar, se extiende una mano y se pasa por su contorno el dedo índice de la otra, recorriendo los dedos uno a uno, mientras se recrean en la caricia. Se repite cambiando de mano. Se hacen tres respiraciones profundas para cerrar la actividad. Hacer esta fase final en parejas es otra opción. Puede observarse esta actividad y otras similares en:

https://convivencia.wordpress.com/2013/02/25/atencionplena-en-ceip-catalina/

A lo largo de la jornada escolar, existen muchos momentos propicios para trabajar la atención plena. Por ejemplo:

$\checkmark$ Inicio de una sesión de clase de cualquier materia o área.

$\checkmark$ En momentos de alboroto en el aula o ruptura del clima de trabajo.

$\checkmark$ En la transición de una actividad a otra que requiera más concentración.

$\checkmark$ Recuperar la calma tras una actividad muy "movida" (vuelta del recreo, tras la clase de Educación Física o Música, etc.).

Se trata de momentos puntuales, pero sistemáticos, de atención plena que van tomando forma de rutinas y hábitos que el alumnado interiorizará para integrar en su vida cotidiana y constituir un recurso de inestimable valor, una fortaleza interior y una actitud vital que favorezca una vida más consciente y feliz.

\section{LAS FORTALEZAS PERSONALES}

Uno de los aspectos clave de la psicología positiva es el estudio de las fortalezas humanas, que se sitúa dentro de la "teoría de rasgos" y enlaza con la idea de "buen carácter". La investigación sobre los rasgos positivos de la personalidad ha originado una elaborada teoría en torno a las llamadas fortalezas personales (Seligman, 2002; Peterson y Seligman, 2004). Estos autores han creado y validado un modelo descriptivo de la personalidad basado en las fortalezas personales, entendidas como rasgos positivos universales, medibles y educables.

El equipo SATI optó por el modelo de 24 fortalezas de Peterson y Seligman (2004) una vez valorados otros modelos, por considerar que reúne las características siguientes: globalidad, excelente aval teórico, evidencias empíricas y, sobre todo, por su gran aplicabilidad en el mundo educativo.

Un equipo de profesionales liderado por Peterson y Seligman, tras años de investigación, identificó 24 fortalezas personales, agrupadas en torno a seis virtudes generales. Las fortalezas se definen como "estilos moralmente valorables de pensar, sentir y actuar, que contribuyen a una vida en plenitud". Este es el listado (Peterson y Seligman, 2004):

SABIDURÍA Y CONOCIMIENTO: Fortalezas cognitivas que implican la adquisición y el uso del conocimiento.

1. Creatividad.

2. Curiosidad.

3. Apertura mental (juicio, pensamiento crítico).

4. Amor por el aprendizaje.

5. Perspectiva (sabiduría).

CORAJE: Fortalezas emocionales que implican el ejercicio de la voluntad para la consecución de metas ante situaciones de dificultad externa o interna.

6. Valentía.

7. Perseverancia.

8. Integridad.

9. Vitalidad.

HUMANIDAD: Fortalezas interpersonales que implican cuidar y ofrecer amistad y cariño a los demás.

10. Amor.

11. Amabilidad.

12. Inteligencia social (inteligencia emocional, inteligencia personal).

JUSTICIA: Fortalezas cívicas que conllevan una vida en comunidad saludable.

13. Ciudadanía (responsabilidad social, lealtad, trabajo en equipo).

14. Sentido de la justicia.

15. Liderazgo.

MODERACIÓN: Fortalezas que nos protegen contra los excesos.

16. Capacidad de perdonar, misericordia.

17. Modestia, humildad.

18. Prudencia (discreción, cautela).

19. Autocontrol, autorregulación.

TRASCENDENCIA: Fortalezas que forjan conexiones con la inmensidad del universo y proveen de significado a la vida.

20. Apreciación de la belleza y la excelencia.

21. Gratitud.

22. Esperanza (optimismo, proyección hacia el futuro).

23. Sentido del humor.

24. Espiritualidad (sentido religioso, fe, sentido en la vida).

En la bibliografía especializada se puede encontrar más información sobre estas fortalezas humanas. Conviene señalar que cada vez hay más investigaciones que avalan la utilidad de este sistema para potenciar el bienestar y la felicidad en diversos 
campos de la vida humana. El instituto VIA "Values in action" (http://www.viacharacter.org) se crea a iniciativa de Seligman y Mayerson, como un organismo que promueve investigaciones, siendo sus principales herramientas el uso de cuestionarios a través de la red.

Los resultados de las investigaciones han puesto en evidencia la relación entre estas fortalezas y la satisfacción vital, el bienestar psicológico y la felicidad. El desarrollo de competencias relacionadas con las fortalezas humanas actúan como barreras frente a los trastornos psicológicos (Seligman y Csikszentmihalyi, 2000).

La síntesis de algunos de los resultados más destacables en el tema de fortalezas y virtudes relacionados con la educación, se puede consultar en el manual Aulas Felices fruto de la revisión de literatura sobre el tema (Park y Peterson, 2009a, 2009b; Peterson y Park, 2009; Peterson y Seligman, 2004). La educación puede jugar un papel muy importante en el desarrollo de las fortalezas humanas, su impulso y entrenamiento como herramienta para la construcción de la felicidad.

Respecto a la fortaleza $\mathrm{n}^{\circ}$ 12, inteligencia social o emocional, es imprescindible abordarla desde el punto de vista educativo. Para la realización de la propuesta de actividades en torno a esta fortaleza se tuvo en cuenta las aportaciones de diferentes autores como Fernández Berrocal, Salovey y Mayer, y el material elaborado y graduado por edades elaborado por el GROP. Esta fortaleza se relaciona con otras como ciudadanía, autocontrol o apertura mental.

Insertar el modelo de fortalezas de Peterson y Seligman en el currículum aporta un marco integrador, que permite abordar el trabajo escolar en torno a las tres competencias clave de carácter más transversal: sentido de iniciativa y espíritu emprendedor, competencia social y cívica y competencia para aprender a aprender.

A pesar de su importancia y del respaldo normativo, estas competencias corren el riesgo de quedar ausentes de las aulas por no corresponderse exactamente con áreas curriculares tradicionales. El equipo SATI relaciona las 24 fortalezas personales con dichas competencias que pueden considerarse como un desglose operativo de ellas. En la tabla 1 se concreta dicha relación.

\section{ESTRATEGIAS DE INTERVENCIÓN}

Para trabajar cada una de las fortalezas personales, en el programa "Aulas felices" se pueden encontrar más de trescientas actividades dirigidas al alumnado de educación infantil, primaria y secundaria. Estas actividades se acompañan de una serie de estrategias de intervención, que son complementarias entre sí: propuestas globales, propuestas específicas, planes personalizados y trabajo conjunto entre familias y centros.

1. Propuestas globales.- Se trata de los cinco principios siguientes para crear ambiente positivo en las escuelas, potenciar el bienestar y el aprendizaje, y favorecer el desarrollo de las fortalezas personales. $\checkmark$ La actitud del profesorado es muy importante. Alude, entre otros, al papel de los adultos como modelo y referente en el aula, concretamente en el trabajo de las fortalezas personales. El impulso de los rasgos positivos en el alumnado debe ir acompañado por el trabajo del docente. El esfuerzo y coherencia del docente será la invitación y activación de la respuesta del alumnado.

$\checkmark$ Crear condiciones de aprendizaje que permitan fluir.

$\checkmark$ Poner en marcha estrategias metodológicas eficaces para aprender y potenciar el bienestar del alumnado,

$\checkmark$ Promover una educación que prime más la calidad que la cantidad.

$\checkmark$ El uso de otros programas complementarios que pueden contribuir al desarrollo de fortalezas personales.

2. Propuestas específicas.- Se trata de una amplia batería de unas 300 actividades dirigidas a trabajar en las aulas las 24 fortalezas personales con el grupo clase y que pueden ser aplicadas por cualquier profesor: tutores y especialistas de las diversas asignaturas. Es aconsejable elegir algunas fortalezas y programar el trabajo de estas en el aula eligiendo las actividades a llevar a cabo para no saturarse ni agobiarse y hacer de los principios anteriores una constante que impregne el trabajo en el aula.

Un ejemplo es la actividad "mensajes positivos" (de la fortaleza 12 inteligencia social). Su objetivo es transmitir bienestar y felicidad a otras personas a través de mensajes positivos. Este es un resumen de la propuesta: Como motivación e inspiración se visualiza el vídeo "Be happy" (puede localizarse en YouTube: http://www.youtube.com/watch?v=SpbMt7ehgeo). Des-

\begin{tabular}{|c|c|c|c|}
\hline \multicolumn{4}{|c|}{ TABLA 1} \\
\hline Competencias & INICIATIVA & SOCIAL Y CIVICA & $\begin{array}{l}\text { APRENDER A } \\
\text { APRENDER }\end{array}$ \\
\hline \multirow{16}{*}{$\begin{array}{l}\text { Fortalezas } \\
\text { Personales }\end{array}$} & Creatividad & Perspectiva & Creatividad \\
\hline & Curiosidad & Integridad & Curiosidad \\
\hline & Apertura mental & Amor & Apertura mental \\
\hline & Amor por el & Amabilidad & Amor por el \\
\hline & aprendizaje & Inteligencia social & aprendizaje \\
\hline & Valentía & Ciudadanía & Perspectiva \\
\hline & Perseverancia & Sentido de la justicia & Perseverancia \\
\hline & Vitalidad & Liderazgo & Ciudadanía \\
\hline & Modestia & Capacidad de & Autocontrol \\
\hline & Prudencia & perdonar & \\
\hline & Autocontrol & Gratitud & \\
\hline & $\begin{array}{l}\text { Apreciación de la } \\
\text { belleza }\end{array}$ & Modestia & \\
\hline & Esperanza & & \\
\hline & Sentido del humor & & \\
\hline & Espiritualidad & & \\
\hline & Liderazgo & & \\
\hline
\end{tabular}


pués se propone al alumnado que redacten mensajes cuya lectura produzca un efecto positivo. Se leen los mensajes (o una muestra de ellos) en clase y se seleccionan algunos para crear carteles que se distribuirán y colocarán por el centro escolar. Se incidirá en la originalidad de los mensajes y que surjan del alumnado. La actividad puede trabajarse en clase de inglés (u otras lenguas) siguiendo la misma pauta. Ejemplos de mensajes elaborados por alumnado de $4^{\circ}$ de primaria se pueden ver en:

https://convivencia.files.wordpress.com/2015/01/mensaje s-positivos.pdf

https://convivencia.wordpress.com/2015/01/25/buenaspracticas-fortaleza-inteligencia-social-aulas-felices/

3. Planes personalizados.- Se orientan al crecimiento del alumnado tomando en consideración las características diferenciales de cada persona y su perfil concreto de fortalezas personales. En este nivel el alumnado pasa el cuestionario VIA y elige las fortalezas que va a ejercitar marcándose un plan de trabajo individual.

4. Trabajo conjunto entre familias y centros.- Es obvio, pero necesario recordar, que es fundamental informar y formar a las familias para establecer pautas conjuntas de actuación, para favorecer la necesaria coordinación entre familia y escuela en aras de una mayor eficiencia del programa.

\section{¿Y EL PROFESORADO?}

La formación del profesorado es fundamental para cualquier cambio o impulso de mejora educativa. El modelo que impulsa el equipo SATI apuesta por una formación teórica y práctica, con ejemplos de buenas propuestas de aula en diferentes etapas educativas, complementado con un compromiso personal de trabajo en el aula. Incluye vivenciar en primera persona las fortalezas y la atención plena, con la pauta de ejercitación entre sesiones. Actividades del programa Aulas Felices en diferentes centros educativos están disponibles para el profesorado en este enlace: https://convivencia.wordpress.com/2013/06/06/9525/

Como síntesis podemos afirmar que la educación es mucho más que la instrucción en los contenidos académicos tradicionales. Una auténtica educación debe impulsar el desarrollo personal y social de todo el alumnado. La finalidad última de la educación es la promoción del bienestar personal y social.

En los apartados anteriores se pretende transmitir que aprendizaje y bienestar son compatibles. Programas de educación emocional, el programa Aulas Felices y otras propuestas son importantes y necesarias para impulsar el desarrollo personal y social, así como el bienestar del alumnado.

Es crucial que dichos programas no solo aporten propuestas de actividades adecuadas a nuestro contexto educativo, sino que deben acompañarse de sugerencias metodológicas y criterios de actuación, que deben impregnar el trabajo diario de aula. Para ser efectivos se debería proceder a una integración curricular siempre que sea posible. Es importante que las intervenciones docentes no sean actuaciones aisladas, sino un continuo que impregna todas las tareas del aula. Esto implica que las acciones programadas emanen de los documentos de centro (PEC, PCE, PAT). Es decir, que estos documentos recojan entre sus objetivos y actividades la educación emocional y el bienestar. Y que estas prácticas y su esencia traspasen los muros del centro y lleguen a toda la comunidad educativa (profesorado, alumnado, personal no docente, familias, entorno), beneficiándose todos de sus efectos positivos. Esto requiere como punto de partida la sensibilización y formación del profesorado, y es fundamental que la formación sea impulsada por la Administración pública.

\section{REFERENCIAS}

Agulló Morera, M. J., Filella Guiu, G., García Navarro, E., López Cassà, E., Bisquerra Alzina, R. (Ed.), (2010). La educación emocional en la práctica. Barcelona: Horsori-ICE.

Álvarez, M. (2001). Diseño y evaluación de programas de educación emocional. Barcelona: Wolters-Kluwer.

Arguís, R. (2014). Mindfulness y educación: aprendiendo a vivir con atención plena. En A. Cebolla, J. García Campayo y M. Demarzo (Eds.), Mindfulness y ciencia (pp. 236-265). Madrid: Alianza.

Bisquerra, R. (2000). Educación emocional y bienestar. Barcelona: Wolters Kluwer-Praxis.

Bisquerra, R. (2008). Educación para la ciudadanía. El enfoque de la educación emocional. Barcelona: Wolters Kluwer.

Bisquerra, R. (2009). Psicopedagogía de las emociones. Madrid: Síntesis.

Bisquerra, R. (2012). Orientación, tutoría y educación emocional. Madrid: Síntesis.

Bisquerra, R. (2013). Cuestiones sobre bienestar. Madrid: Síntesis.

Bisquerra, R. (2015). Universo de emociones. Valencia: PalauGea.

Bisquerra, R. (Coord.) (2011). Educación emocional. Propuestas para educadores y familias. Bilbao: Desclée de Brower.

Bisquerra, R. (Coord.). (2014). Prevención del acoso escolar con educación emocional. Bilbao: Desclée de Brower.

Bisquerra, R., Pérez-González, J. C., y García Navarro, E. (2014). Inteligencia emocional en la educación. Madrid: Síntesis.

Brown, K. W., y Ryan, R. M. (2003). The Benefits of Being Present: Mindfulness and Its Role in Psychological Well-Being. Journal of Personality and Social Psychology, 84, 4, 822848.

Cebolla, A., y Miró, M. T. (2008). Efectos de la Terapia Cognitiva basada en la Atención Plena: una aproximación cualitativa. Apuntes de Psicología, 26, 2, 257-268.

Davidson, R. J., y Begley, S. (2012). The Emotional Life of your Brain. Londres: Hodder \& Stoughton. 
Equipo SATI. (2012). Programa Aulas Felices. Psicología Positiva aplicada a la Educación. www. aulasfelices.com, www. aulasfelices.org

Filella, G. (2014). Aprendre a conviure. Barcelona: Barcanova.

GROP (2009). Actividades para el desarrollo de la inteligencia emocional en los niños. Barcelona: Parramón.

Güell, M., y Muñoz, J. (Coord.), (2003). Educación emocional. Programa para la educación secundaria postobligatoria. Barcelona: Wolters Kluwer.

Kabat-Zinn, J. (1990). Vivir con plenitud las crisis. Como utilizar la sabiduría del cuerpo y de la mente para afrontar el estrés, el dolor y la enfermedad. Barcelona: Kairós.

Kabat-Zinn, J. (2005). La práctica de la atención plena. Barcelona: Kairós (2007).

Lavilla, M., Molina, D. y López, B. (2008). Mindfulness. O cómo practicar el aquí y el ahora. Barcelona: Paidós.

Linley, P. A., Stephen, J., Harrington, S. y Wood, A. M. (2006). Positive psychology: Past, present, and (possible) future. The Journal of Positive Psychology, 1, 3-16.

López Cassà, E. (2003). Educación emocional. Programa para 3-6 años. Barcelona: Praxis.

López Cassà, È. (2011). Educar las emociones en la infancia (de 0 a 6 años). Reflexiones y propuestas prácticas. Madrid: Wolters Kluwer.

Lyubomirsky, S. (2008). La ciencia de la felicidad. Barcelona: Urano.

Mayer, J. D., Salovey, P., Caruso, D. R., y Cherkasskiy, L. (2015). La inteligencia emocional. En Fundación Botín, De la neurona a la felicidad. Diez propuestas desde la inteligencia emocional (pp. 9-32). Santander: Fundación Botín.

Pascual, V., y Cuadrado, M. (2001). Educación emocional. Programa de actividades para educación secundaria obligatoria. Barcelona: Praxis.

Peterson, C., y Park, N. (2009). El estudio científico de las for- talezas humanas. En C. Vázquez, C., y G. Hervás. (Eds.), La ciencia del bienestar (pp. 181-207). Madrid: Alianza.

Peterson, Ch. y Seligman, M. E. P. (2004). Character Strengths and Virtues: A Handbook and Classification. Washington: Oxford University Press.

Peterson, Ch., Park, N., y Seligman, M. E. P. (2005). Orientations to happiness and life satisfaction: the full life versus the empty life. Journal of Happiness Studies, 6, 25-41.

Pujol, E., y Bisquerra, R. (2012). El gran libro de las emociones. Barcelona: Parramón.

Redorta, J., Obiols, M., y Bisquerra, R. (2006). Emoción y conflicto. Aprenda a manejar las emociones. Barcelona: Paidós.

Renom, A. (2003). Educación emocional. Programa para la educación primaria. Barcelona: Praxis.

Salovey, P., y Mayer, J. D. (1990). Emotional Intelligence. Imagination, Cognition, and Personality, 9, 185-211.

Seligman, M. E. P. (2003). La auténtica felicidad. Barcelona: Ediciones $B$.

Seligman, M. E. P. (2011). Flourish: A Visionary New Understanding of Happiness and wellbeing. New York: Free Press.

Seligman, M. E. P., Steen, T. A., Park, N., y Peterson, Ch. (2005). Empirical validation of interventions. American Psychologist, 60, 5, 410-421.

Seligman, M. E. P., y Csikszentmihalyi, M. (2000). Positive Psychology: An Introduction. American Psychologist, 55, 1, 5-14.

Simón, V. M. (2007). Mindfulness y Neurobiología. Revista de Psicoterapia, 17, 66/67, 5-30.

Soldevila, A (2009). Emociónate. Programa de educación emocional. Madrid: Pirámide.

Vallejo Pareja, M. A. (2006). Mindfulness. Papeles del Psicólogo, 27, 92-99.

Vázquez, C, y Hervás, G. (Eds.), (2008). Psicología positiva aplicada. Bilbao: Desclée de Brouwer. 